\title{
An Analysis of the Predictors of Major Bleeding After Transcatheter Aortic Valve Transplantation Using the National Inpatient Sample (2015-2018)
}

Henna Khan ${ }^{1}$, Asma Gilani ${ }^{1}$, Ihtisham Qayum ${ }^{2}$, Taif Khattak ${ }^{3}$, Furqan Haq ${ }^{4}$, Muhammad Zahid Anwar ${ }^{4}$ , Muhammad Atif Khan ${ }^{5}$, Sayyed Jalawan Asjad ${ }^{6}$, Sakina Abbas ${ }^{7}$, Arslan Inayat ${ }^{8}$

1. Medicine, Khyber Girls Medical College, Peshawar, PAK 2. Internal Medicine, Khyber Teaching Hospital Peshawar, Peshawar, PAK 3. Emergency Medicine, Hamad General Hospital, Doha, QAT 4. Medicine, Khyber Medical College, Peshawar, PAK 5. Internal Medicine, Texas Tech University Health Sciences Center, Amarillo, USA 6. Internal Medicine, Royal Preston Hospital, Fulwood, GBR 7. Medicine, Dow University of Health Sciences, Karachi, PAK 8. Internal Medicine, University at Buffalo, Catholic Health System, Buffalo, USA

Corresponding author: Arslan Inayat, arslaninayat88@gmail.com

\section{Abstract}

\section{Background}

Transcatheter aortic valve replacement (TAVR) is now a common procedure to treat and improve quality of life, clinical outcomes, and self-sufficiency in high-risk patients with aortic stenosis, and its use has been expanding rapidly in younger and low-risk populations. The aim of this study was to evaluate the outcomes, trends, and predictors of major bleeding in patients undergoing TAVR.

\section{Methodology}

We utilized the National Inpatient Sample (NIS) data from the year 2015 to 2018. International Classification of Disease 10 codes were utilized to extract data. Baseline characteristics were compared using Pearson's chisquare test for categorical variables and independent samples t-test for continuous variables. A multivariable logistic regression model was used to evaluate the predictors of major bleeding. Propensity matching was done for adjusted analysis to compare outcomes in TAVR with and without major bleeding. The outcomes of interest in this study were (1) predictors of major bleeding after TAVR; (2) in-hospital mortality; and (3) resource utilization in terms of cost and length of stay.

\section{Results}

A total of 34,752 weighted hospitalizations for TAVR were included in the analysis. Of the patients undergoing the procedure, 2,294 (6.6\%) had a major bleed while 32,458 (93.3\%) did not. At baseline, patients with coagulopathy (odds ratio [OR]: 2.03 ; $95 \%$ confidence interval [CI]: 1.82-2.27), congestive heart failure (OR: 1.26; 95\% CI: 1.13-1.40), chronic obstructive pulmonary disease (OR: 1.41; 95\% CI: 1.29-1.55), liver disease (OR: 1.96; 95\% CI: 1.61-2.39), peripheral vascular disease (OR: 1.29; 95\% CI: 1.17-1.43), cerebrovascular disease (OR: 1.22; 95\% CI: 1.07-1.38), end-stage renal disease (ESRD) (OR: 2.17; 95\% CI: 1.82-2.59), and coronary artery disease (OR: $1.17 ; 95 \% \mathrm{Cl}: 1.06-1.30$ ) had higher adjusted rates of odds of

Review began 06/07/2021 Review ended 06/21/2021 Published 06/29/2021

\section{() Copyright 2021}

Khan et al. This is an open access article distributed under the terms of the Creative Commons Attribution License CC-BY 4.0., which permits unrestricted use, distribution, and reproduction in any medium, provided the original author and source are credited. major bleeding. Patients who had major bleeding had a higher median cost of stay (US\$60,326 vs. US\$45490) and length of stay (seven vs. three days).

\section{Conclusions}

Mortality is higher in patients with major bleeding, and at baseline, coagulopathy and ESRD are significant predictors of a major bleed in patients undergoing TAVR.

Categories: Cardiac/Thoracic/Vascular Surgery, Cardiology, Internal Medicine

Keywords: transcatheter aortic valve implantation, transcatheter aortic valve replacement, tavr, tavi, aortic stenosis, major bleeding

\section{Introduction}

In the medium-to-high-risk elderly patients who suffer from severe aortic stenosis and calcified aortic valve disease, transcatheter aortic valve replacement (TAVR) has proven to be an effective and minimally invasive procedure [1,2]. The three landmark PARTNER trials have exhaustively evaluated the efficacy and side effect profile of TAVR which has stood the test of time [2,3]. It is an effective alternative to surgical valve replacement and has been widely adopted in the United States [3,4].

According to the Society of Thoracic Surgeons/American College of Cardiology Transcatheter Valve Therapy Registry investigators, TAVR has been reported to have a $92 \%$ procedural success rate [5]. In patients with aortic stenosis, TAVR has led to constant improvement in clinical outcomes along with improved techniques 
Major bleeding is a life-threatening complication of surgical aortic valve replacement (10\% surgical aortic valve replacement [SAVR] versus $6 \%$ for TAVR) [1]. However, it is important to mention that despite complication rates being lower with TAVR compared to the surgical option, major bleeding remains a significant complication identified in the landmark PARTNER trials [1-3].

One of the most frequent complications post-TAVR with the first PARTNER trial was major bleeding which reported $10.2 \%$ bleeding events [8]. Major bleed is known to be associated with significant mortality and morbidity even though it is less frequent compared to SAVR $[9,10]$. Post-TAVR, major bleeding was reported to increase one-month mortality by $32 \%$ [11]. Data on outcomes of TAVR patients who develop major bleeding remain limited. Hence, the main objective of this study was to comprehensively evaluate the predictors and outcomes of major bleeding in TAVR using the National Inpatient Sample (NIS) database.

\section{Materials And Methods}

The NIS database was used to identify cases of TAVR performed during 2015-2018. NIS is a publicly available database and does not contain any patient-sensitive information. Hence, this study did not require ethical board approval or informed consent [12]. NIS data are collected annually and are representative of $20 \%$ of the hospitalizations in the United States. The cost of hospitalization is also recorded in US dollars which comprises the cost incurred in return for services provided.

National analysis was performed using the international classification of diseases (ICD)-9 (3505, 3506), and ICD-10 (02RF3) codes were utilized to identify all cases of TAVR. We extracted cases from all available procedure fields. Patients younger than 18 years of age were not included in the analysis. Data on comorbidities are provided by the NIS. Major bleeding in our analysis was defined as any bleeding requiring transfusion. The outcomes of interest for our study were (1) predictors of major bleeding after TAVR; (2) inhospital mortality; and (3) resource utilization in terms of cost and length of stay.

We used the discharge weights provided by NIS to perform analysis on weighted hospitalizations. We used the Mann-Whitney U test for all continuous variables as they are not normally distributed, and the chisquare test was used for categorical variables. To test for the non-normality of data we used the ShapiroWilk test. Entry method was used to develop a binary logistic model including baseline comorbidities such as obesity, weight loss, metastatic cancer, lymphoma, solid organ tumor, alcohol use, coagulopathy, hypothyroidism, chronic obstructive pulmonary disease (COPD), cerebrovascular disease (CVA), congestive heart failure (CHF), coronary artery disease (CAD), diabetes mellitus, hypertension, liver disease, chronic kidney disease (CKD), and peripheral vascular disease (PVD). Demographic factors such as age, sex, race, median income, and hospital location were also included in the analysis. Observations with less than 11 cases were not reported in compliance with the Health Cost and Utilization Project. R software version 3.5 was used for all analyses. We considered a p-value of $<0.05$ to be statistically significant.

\section{Results}

A total of 34,752 weighted hospitalizations for TAVR were included in the analysis. Of the patients undergoing the procedure, 2,294 (6.6\%) had bleeding complications while 32,458 (93.3\%) did not. The detailed baseline characteristics are summarized in Table 1 . At baseline, patients with coagulopathy (odds ratio [OR]: 2.03; 95\% confidence interval [CI]: 1.82-2.27), CHF (OR: 1.26; 95\% CI: 1.13-1.40), COPD (OR: 1.41; 95\% CI: 1.29-1.55), liver disease (OR: 1.96; 95\% CI: 1.61-2.39), PVD (OR: 1.29; 95\% CI: 1.17-1.43), CVA (OR: 1.22; 95\% CI: 1.07-1.38), end-stage renal disease (ESRD) (OR: 2.17; 95\% CI: 1.82-2.59), and CAD (OR: 1.17 ; $95 \% \mathrm{Cl}: 1.06-1.30$ ) had higher adjusted rates of odds of major bleeding (Table1; Figure 1). Patients who had major bleeding had a higher median cost of stay (US $\$ 60,326$ vs. US $\$ 45,490$ ) and length of stay (seven vs. three days) (Table 2). 


\section{Cureus}

\begin{tabular}{|c|c|c|c|}
\hline & & & Multivariate analysis OR (95\% Cl) \\
\hline Variable. (\%) & Without major bleed $(32,458)$ & With major bleed $(2,294)$ & No bleeding vs. bleeding \\
\hline Age, median (IQR) & $81(75-86)$ & $82(76-87)$ & $1.37(1.22-1.53)$ \\
\hline Female gender & $14,823(45.7 \%)$ & $1,290(56.2 \%)$ & $1.69(1.54-1.85)$ \\
\hline Caucasian & 27,107 (87.2\%) & $1,828(82.6 \%)$ & Reference \\
\hline African Americans & $1,267(4.1 \%)$ & $146(6.6 \%)$ & $1.53(1.27-1.86)$ \\
\hline Hispanics & $1,480(4.8 \%)$ & 148 (6.7\%) & $1.48(1.24-1.78)$ \\
\hline Alcohol use & $52(0.2 \%)$ & $5(0.2 \%)$ & $1.02(0.39-2.62)$ \\
\hline Hypothyroidism & 6,591 (20.3\%) & 489 (21.3\%) & $0.96(0.86-1.07)$ \\
\hline Coagulopathy & 3,792 (11.7\%) & 525 (22.9\%) & $2.03(1.82-2.27)$ \\
\hline $\mathrm{CHF}$ & 23,725 (73.1\%) & 1,793 (78.2\%) & $1.26(1.13-1.40)$ \\
\hline CAD & $22,458(69.2 \%)$ & $1,627(70.9 \%)$ & $1.17(1.06-1.30)$ \\
\hline Cerebrovascular disease & 3,711 (11.4\%) & 339 (14.8\%) & $1.22(1.07-1.38)$ \\
\hline COPD & $9,785(30.1 \%)$ & $863(37.6 \%)$ & $1.41(1.29-1.55)$ \\
\hline Diabetes mellitus & $5,144(15.8 \%)$ & $282(12.3 \%)$ & $0.85(0.75-0.98)$ \\
\hline Hypertension & $28,886(89.0 \%)$ & 2,038 (88.8\%) & $0.90(0.78-1.03)$ \\
\hline Liver disease & $949(2.9 \%)$ & $144(6.3 \%)$ & $1.96(1.61-2.39)$ \\
\hline ESRD & $1,128(3.5 \%)$ & $186(8.1 \%)$ & $2.17(1.82-2.59)$ \\
\hline Obesity & 5,545 (17.1\%) & $296(12.9 \%)$ & $0.81(0.71-0.92)$ \\
\hline PVD & $7,000(21.6 \%)$ & $629(27.4 \%)$ & $1.29(1.17-1.43)$ \\
\hline Weight loss & $979(3.0 \%)$ & $168(7.3 \%)$ & $2.09(1.74-2.50)$ \\
\hline Metastatic cancer & $210(0.6 \%)$ & $19(0.8 \%)$ & $1.20(0.71-2.03)$ \\
\hline Lymphoma & $221(0.7 \%)$ & $16(0.7 \%)$ & $1.21(0.72-2.03)$ \\
\hline Solid organ tumor & $780(2.4 \%)$ & $72(3.1 \%)$ & $1.29(0.98-1.69)$ \\
\hline Income $0-25^{\text {th }}$ percentile & 6,838 (21.4\%) & $481(21.3 \%)$ & Reference \\
\hline $25^{\text {th }}-50^{\text {th }}$ & $8,255(25.8 \%)$ & $493(21.8 \%)$ & $0.92(0.80-1.05)$ \\
\hline $50^{\text {th }}-75^{\text {th }}$ & $8,544(26.7 \%)$ & $608(26.9 \%)$ & $1.11(0.98-1.27)$ \\
\hline $75^{\text {th }}-100^{\text {th }}$ & $8,336(26.1 \%)$ & $677(30.0 \%)$ & $1.26(1.11-1.44)$ \\
\hline Urban & $277(0.9 \%)$ & $21(0.9 \%)$ & Reference \\
\hline Urban nonteaching & $2,997(9.2 \%)$ & $238(10.4 \%)$ & $1.09(0.66-1.81)$ \\
\hline Urban teaching & 29,184 (89.9\%) & 2,035 (88.7\%) & $0.91(0.56-1.48)$ \\
\hline
\end{tabular}

\section{TABLE 1: Baseline characteristics and predictors of major bleeding in patients after TAVR.}

OR: odds ratio; CI: confidence interval; IQR: interquartile range; CHF: congestive heart failure; CAD: coronary artery disease; COPD: chronic obstructive pulmonary disease; ESRD: end-stage renal disease; PVD: peripheral vascular disease; TAVR: transcatheter aortic valve replacement 


\section{Cureus}

\begin{tabular}{|c|c|c|c|}
\hline Outcome & No bleed & Bleed & P-value \\
\hline Died during hospitalization & $432(1.3 \%)$ & $146(6.4 \%)$ & $<0.01$ \\
\hline Median length of stay (days) & $3(2-5)$ & $7(4-13)$ & $<0.01$ \\
\hline Median cost of stay (US\$) & $45,490(35,640-57,665)$ & 60,326 $(45,756-81,222)$ & $<0.01$ \\
\hline
\end{tabular}

\section{TABLE 2: In-hospital outcomes of patients with and without bleed in TAVR.}

TAVR: transcatheter aortic valve replacement



\section{Discussion}

Approximately $6 \%$ of patients who undergo TAVR have major bleeding. Of the patients who have major bleeding, 6.4\% die during hospitalization. We also identified important baseline characteristics such as age greater than 75, female sex, and history of ESRD, liver disease, PVD, CHF, and CAD.

Major bleeding or vascular complications are expected to decrease as TAVR technology evolves into smaller device sizes $[13,14]$. However, bleeding complications have been underreported and are inconsistent in the early literature [10]. Patients who undergo TAVR are typically frail, elderly, and are at a risk for both bleeding and ischemic complications $[12,15,16]$. Careful risk and benefit evaluation is warranted to identify the antithrombotic regimen, as major late bleeding complications are not only frequent but also associated with an increased risk of total mortality [17]. With baseline hematological problems, higher bleeding complications related to coagulation factors and platelets were identified in our study. According to the literature, our findings are reinforced by a good association between blood disorders and major bleeding events [16].

According to a previous study, major bleeding is associated with a three-fold increase in one-year mortality following TAVR and SAVR [18]. A previous study reported that major bleeding and life-threatening bleeding after TAVR, as defined by the Valve Academic Research Consortium (VARC) criteria, occurred in 
approximately 15-20\% of TAVR procedures [13]. Factors that increase the risk of bleeding include a high prevalence of $\mathrm{CKD}$, peripheral vasculopathy, acquired thrombocytopenia, and acquired reversible von Willebrand factor deficiency [16,19-21]. It is important to note that our definition of major bleeding was not based on well-validated VARC-II criteria similar to previous studies, instead, we used ICD codes to define major bleeding. However, our study findings are in agreement with prior reported data as we report higher bleeding risk with PVD (1.29 times) and coagulopathy (2.03 times).

After the procedure, patients undergoing TAVR had lower rates of major or life-threatening bleeding (11.3\% vs. 20.9\%), acute kidney injury (AKI) stage II and III, and cardiogenic shock compared to those undergoing SAVR [13,20-26]. Due to the inherent platelet dysfunction, patients with ESRD have a propensity to bleed and are associated with high mortality $[19,20]$. Similarly, due to associated coagulopathy, liver disease patients have an increased tendency to bleed [27]. In a previous meta-analysis, patients with chronic liver disease had a higher incidence of bleeding complications, need for blood transfusions, and mortality, which was further exacerbated by antiplatelet drug use [28,29]. As reported by Tchetche et al., $38.9 \%$ of patients undergoing TAVR received at least one transfusion [10,13,19,29]. Our estimate of major bleeding complications in TAVR was $6.6 \%$, which we believe to be a more contemporary estimate. Moreover, we report 2.17 and 1.96 times higher odds of bleeding with ESRD and liver disease, respectively. Interestingly, our study found that obesity and hypertension are associated with a lower risk of bleeding. The phenomenon of the obesity paradox has been seen in our study, which was previously described for diseases such as myocardial infarction, heart failure, and renal disease [30]. Similarly, prior TAVR literature data based on NIS reported hypertension as a protective factor against mortality [18].

NIS provides an opportunity to identify characteristics from a large sample size to evaluate the predictors. For instance, previously, it was found that peptic ulcer disease and colon cancer lead to high gastrointestinal bleeding after TAVR. This led to a change in the recommendation to perform a pre-TAVR colonoscopy and proton pump inhibitor use to mitigate the risk. Similarly, patients undergoing TAVR who were found to have a high risk of AKI underwent pre-TAVR intravenous hydration along with minimal contrast use during the procedure. Our study aimed to replicate this by providing data on these predictors so that mitigation measures can be taken against bleeding.

Our study findings are not without limitations. For instance, we could not extract data on antiplatelet and anticoagulant use as they are not available in the NIS database. Similarly, data on the cause of death are not available in the NIS. Moreover, NIS coding errors cannot be completely ruled out.

\section{Conclusions}

We report that at baseline, ESRD, liver disease, PVD, CHF, CAD, age greater than 75, and female gender are associated with a higher risk of bleeding after TAVR. It is of utmost importance to identify patients who are at a high risk of developing complications as TAVR expands to a rapidly aging population.

\section{Additional Information \\ Disclosures}

Human subjects: All authors have confirmed that this study did not involve human participants or tissue. Animal subjects: All authors have confirmed that this study did not involve animal subjects or tissue. Conflicts of interest: In compliance with the ICMJE uniform disclosure form, all authors declare the following: Payment/services info: All authors have declared that no financial support was received from any organization for the submitted work. Financial relationships: All authors have declared that they have no financial relationships at present or within the previous three years with any organizations that might have an interest in the submitted work. Other relationships: All authors have declared that there are no other relationships or activities that could appear to have influenced the submitted work.

\section{References}

1. Rotman OM, Bianchi M, Ghosh RP, Kovarovic B, Bluestein D: Principles of TAVR valve design, modelling, and testing. Expert Rev Med Devices. 2018, 15:771-91. 10.1080/17434440.2018.1536427

2. Mack MJ, Leon MB, Thourani VH, et al.: Transcatheter aortic-valve replacement with a balloon-expandable valve in low-risk patients. N Engl J Med. 2019, 380:1695-705. 10.1056/NEJMoa1814052

3. Otto CM, Nishimura RA, Bonow RO, et al.: 2020 ACC/AHA guideline for the management of patients with valvular heart disease: executive summary: a report of the American College of Cardiology/American Heart Association Joint Committee on Clinical Practice Guidelines. Circulation. 2021, 143:e35-71. 10.1161/CIR.0000000000000932

4. Ullah W, Sattar Y, Al-Khadra Y, et al.: Clinical outcomes of renal and liver transplant patients undergoing transcatheter aortic valve replacement: analysis of national inpatient sample database. Expert Rev Cardiovasc Ther. 2021, 19:363-8. 10.1080/14779072.2021.1892489

5. Zaleska-Kociecka M, Dabrowski M, Stepinska J: Acute kidney injury after transcatheter aortic valve replacement in the elderly: outcomes and risk management. Clin Interv Aging. 2019, 14:195-201. 10.2147/CIA.S149916

6. Tchetche D, de Biase C, Brochado B, Mastrokostopoulos A: How to make the TAVI pathway more efficient. Interv Cardiol. 2019, 14:31-3. 10.15420/icr.2018.28.2 
7. Ullah W, Saleem S, Sattar Y, et al.: Abstract 17383: comparative analysis of TAVR with PCI and SAVR with CABG - a Nationwide Inpatient Sample database. Circulation. 2020, 142:17383.

10.1161/circ.142.suppl_3.17383

8. Smith CR, Leon MB, Mack MJ, et al.: Transcatheter versus surgical aortic-valve replacement in high-risk patients. N Engl J Med. 2011, 364:2187-98. 10.1056/NEJMoa1103510

9. Tchetche D, Van der Boon RM, Dumonteil N, et al.: Adverse impact of bleeding and transfusion on the outcome post-transcatheter aortic valve implantation: insights from the Pooled-RotterdAm-MilanoToulouse In Collaboration Plus (PRAGMATIC Plus) initiative. Am Heart J. 2012, 164:402-9. 10.1016/j.ahj.2012.07.003

10. Généreux P, Head SJ, Van Mieghem NM, et al.: Clinical outcomes after transcatheter aortic valve replacement using valve academic research consortium definitions: a weighted meta-analysis of 3,519 patients from 16 studies. J Am Coll Cardiol. 2012, 59:2317-26. 10.1016/j.jacc.2012.02.022

11. Wang J, Yu W, Jin Q, Li Y, Liu N, Hou X, Yu Y: Risk factors for post-TAVI bleeding according to the VARC-2 bleeding definition and effect of the bleeding on short-term mortality: a meta-analysis. Can J Cardiol. 2017, 33:525-34. 10.1016/j.cjca.2016.12.001

12. Overview of the National (Nationwide) Inpatient Sample (NIS) . (2019). Accessed: January 20, 2021: https://www.hcup-us.ahrq.gov/nisoverview.jsp\#about.

13. Kolte D, Vlahakes GJ, Palacios IF, Sakhuja R, Passeri JJ, Inglessis I, Elmariah S: Transcatheter versus surgical aortic valve replacement in low-risk patients. J Am Coll Cardiol. 2019, 74:1532-40. 10.1016/j.jacc.2019.06.076

14. Dangas GD, Tijssen JGP, Wöhrle J, et al.: A controlled trial of rivaroxaban after transcatheter aortic-valve replacement. N Engl J Med. 2020, 382:120-9. 10.1056/NEJMoa1911425

15. Mangieri A, Montalto C, Poletti E, et al.: Thrombotic versus bleeding risk after transcatheter aortic valve replacement: JACC review topic of the week. J Am Coll Cardiol. 2019, 74:2088-101. 10.1016/j.jacc.2019.08.1032

16. Ferlini M, Mauri S, Rossini R: Dual antiplatelet therapy after TAVR: a drop in the bucket? . Int J Cardiol. 2019, 280:46-8. 10.1016/j.ijcard.2019.01.069

17. Bendayan M, Messas N, Perrault LP, et al.: Frailty and bleeding in older adults undergoing TAVR or SAVR: insights from the FRAILTY-AVR study. JACC Cardiovasc Interv. 2020, 13:1058-68. 10.1016/j.jcin.2020.01.238

18. Ullah W, Jafar M, Zahid S, et al.: Predictors of in-hospital mortality in patients with end-stage renal disease undergoing transcatheter aortic valve replacement: A nationwide inpatient sample database analysis [In Press]. Cardiovasc Revasc Med. 2021, 10.1016/j.carrev.2021.02.002

19. Ullah W, Zahid S, Hamzeh I, Birnbaum Y, Virani SS, Alam M: Trends and predictors of transcatheter aortic valve implantation related in-hospital mortality (From the National Inpatient Sample Database). Am J Cardiol. 2021, 143:97-103. 10.1016/j.amjcard.2020.12.031

20. Zahid S, Ullah W, Khan MU, Salama A, Krupica T, Khan MZ: Predictors of acute kidney injury after transcatheter aortic valve implantation (From National Inpatient Sample [2011-2018]) [In Press]. Am J Cardiol. 2021, 10.1016/j.amjcard.2021.04.003

21. Zhang X, Wang T, Lan R, et al.: Meta-analysis comparing results of transcatheter versus surgical aorticvalve replacement in patients with severe aortic stenosis. Am J Cardiol. 2020, 125:449-58. 10.1016/j.amjcard.2019.10.057

22. Fu J, Popal MS, Li Y, et al.: Transcatheter versus surgical aortic valve replacement in low and intermediate risk patients with severe aortic stenosis: systematic review and meta-analysis of randomized controlled trials and propensity score matching observational studies. J Thorac Dis. 2019, 11:1945-62. 10.21037/jtd.2019.04.97

23. Mc Morrow R, Kriza C, Urbán P, et al.: Assessing the safety and efficacy of TAVR compared to SAVR in lowto-intermediate surgical risk patients with aortic valve stenosis: an overview of reviews. Int J Cardiol. 2020, 314:43-53. 10.1016/j.ijcard.2020.04.022

24. Witberg G, Lador A, Yahav D, Kornowski R: Transcatheter versus surgical aortic valve replacement in patients at low surgical risk: a meta-analysis of randomized trials and propensity score matched observational studies. Catheter Cardiovasc Interv. 2018, 92:408-16. 10.1002/ccd.27518

25. Ullah W, Zahid S, Zaidi R, et al.: Predictors of permanent pacemaker implantation in patients undergoing transcatheter aortic valve replacement - a systematic review and meta-analysis. J Am Coll Cardiol. 2021, 77:981. 10.1016/S0735-1097(21)02340-8

26. Thyregod HG, Steinbrüchel DA, Ihlemann N, et al.: Transcatheter versus surgical aortic valve replacement in patients with severe aortic valve stenosis: 1-year results from the all-comers NOTION randomized clinical trial. J Am Coll Cardiol. 2015, 65:2184-94. 10.1016/j.jacc.2015.03.014

27. Lambert MP: Platelets in liver and renal disease . Hematology Am Soc Hematol Educ Program. 2016, 2016:251-5. 10.1182/asheducation-2016.1.251

28. Ma X, Zhao D, Li J, et al.: Transcatheter aortic valve implantation in the patients with chronic liver disease: a mini-review and meta-analysis. Medicine (Baltimore). 2020, 99:e19766. 10.1097/MD.0000000000019766

29. Durand E, Blanchard D, Chassaing S, et al.: Comparison of two antiplatelet therapy strategies in patients undergoing transcatheter aortic valve implantation. Am J Cardiol. 2014, 113:355-60. 10.1016/j.amjcard.2013.09.033

30. Banack HR, Kaufman JS: The "obesity paradox" explained. Epidemiology. 2013, 24:461-2. 10.1097/EDE.0b013e31828c776c 